\title{
Cahiers de la recherche en éducation
}

\section{L’insertion professionnelle des diplômées et des diplômés universitaires du premier cycle au Québec : hétérogénéité et conséquences}

\section{Mario Charette}

Volume 1, numéro 1, 1994

URI : https://id.erudit.org/iderudit/1018322ar

DOI : https://doi.org/10.7202/1018322ar

Aller au sommaire du numéro

Éditeur(s)

Faculté d'éducation, Université de Sherbrooke

ISSN

1195-5732 (imprimé)

2371-4999 (numérique)

Découvrir la revue

Citer cet article

Charette, M. (1994). L’insertion professionnelle des diplômées et des diplômés universitaires du premier cycle au Québec : hétérogénéité et conséquences. Cahiers de la recherche en éducation, 1(1), 7-36.

https://doi.org/10.7202/1018322ar
Résumé de l'article

L’université québécoise s'est vu confier, durant les années soixante, une mission à double volet : développer une main-d'œuvre hautement qualifiée et permettre la mobilité sociale des individus. Devant les profondes modifications subies présentement par le marché du travail, une remise en question de cette mission s'impose. Cet article présente la position relative sur le marché de l'emploi des diplômées et des diplômés de quatre-vingt-treize programmes du premier cycle, à l'aide de deux indicateurs : l'indice d'emploi à temps plein régulier (IPR) et l'indice d'emploi à temps plein relié au domaine d'études (IPL). Pour chaque indice et chaque programme, les résultats sont regroupés selon la famille de programmes et le type de formation (professionnelle ou non professionnelle). La relation entre les indices et la proportion de diplômées et de diplômés des divers programmes est également examinée. Les résultats conduisent à remettre en question certains des postulats qui ont mené à l'énoncé de mission de l’université québécoise. 
Cahiers de la recherche en éducation, vol. $1, n^{\circ} 1,1994, p .7$ à 36

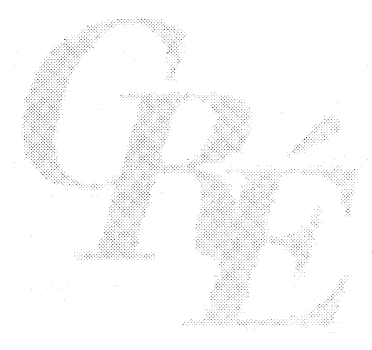

\title{
L'insertion professionnelle des diplômées et des diplômés universitaires du premier cycle au Québec : hétérogénéité et conséquences
}

\author{
Mario Charette
}

Université Laval

\begin{abstract}
Résumé. - L'université québécoise s'est vu confier, durant les années soixante, une mission à double volet : développer une main-d'œuvre hautement qualifiée et permettre la mobilité sociale des individus. Devant les profondes modifications subies présentement par le marché du travail, une remise en question de cette mission s'impose. Cet article présente la position relative sur le marché de l'emploi des diplômées et des diplômés de quatre-vingt-treize programmes du premier cycle, à l'aide de deux indicateurs : l'indice d'emploi à temps plein régulier(IPR) et l'indice d'emploi à temps plein relié au domaine d'études (IPL). Pour chaque indice et chaque programme, les résultats sont regroupés selon la famille de programmes et le type de formation (professionnelle ou non professionnelle). La relation entre les indices et la proportion de diplômées et de diplômés des divers programmes est également examinée. Les résultats conduisent à remettre en question certains des postulats qui ont mené à l'énoncé de mission del'université québécoise.
\end{abstract}




\section{Introduction}

Partout dans le monde industrialisé, la question de l'insertion professionnelle des jeunes a pris une grande importance. De nombreuses études ont fait valoir que ces derniers sont de plus en plus nombreux à éprouver des difficultés à s'insérer dans le marché du travail de leur pays (Coleman et Husén, 1985; Coombs, 1985; Hartmann, 1988; Roberts, 1988). Le Québec n'a malheureusement pas échappé à ce problème. De nombreuses études ont déjà fait valoir que le processus d'entrée sur le marché du travail de la jeunesse québécoise est devenu de plus en plus long et de plus en plus difficile (Tremblay et Van Schendel, 1982; Fortin, 1984; Baril, 1987; Laperrière, 1982). Plus spécifiquement, les jeunes de quinze à vingt-quatre ans demeurent, depuis plus d'une décennie, très nombreux parmi les chômeurs. Depuis 1975, leur taux de chômage n'a jamais diminué en deçà de $13 \%$, et a atteint jusqu'à $23 \%$, et ce, malgré une diminution de leur poids démographique (Tremblay, 1989). De plus, en se basant sur des données de l'Enquête canadienne sur l'activité de 1986, Gauthier (1991) démontre que lorsque ces jeunes détiennent un emploi, fréquemment, ce dernier n'est pas ce qu'elle nomme un emploi «typique», c'est-à-dire un emploi régulier à temps plein, mais bien plutôt un emploi «atypique», c'est-à-dire qu'il est de durée prédéterminée, souvent à temps partiel et qu'il est rémunéré à un salaire moindre que celui de travailleurs plus âgés (Myles, Picott et Wannell, 1988). Ces conditions ont pour effet de garder ces jeunes dans une situation de dépendance sociale qu'on a qualifiée de diverses façons : postadolescence (LeBras, 1983), moratoire social (Erikson, dans Bernier, 1986), «no man's land» économique (Coleman et Husén, 1985).

Ce problème de recherche est encore nouveau au Québec. Il a fallu attendre la récession économique de 1981 et le début d'une remise en question du mythe de la croissance économique continue (Duchastel, 1983) pour que commencent à apparaître des études qui portent sur l'insertion professionnelle des jeunes. De plus, ce n'est que récemment que certaines recherches sont apparues sur le thème plus précis de l'insertion professionnelle des diplômées et des diplômés universitaires (Pageau et Chénard, 1991). Ici comme ailleurs, en effet, les études universitaires ont souvent été décrites comme un moyen efficace d'augmenter ses chances d'obtenir un emploi (Fortin, 1984; Husén, 1989). Une analyse des 
statistiques sur le marché de l'emploi québécois permet d'ailleurs de confirmer que les diplômées et les diplômés universitaires ont généralement un sort meilleur sur le marché de l'emploi que les jeunes du même âge sans diplôme universitaire (Tremblay, 1989; Bureau de la statistique du Québec, 1984). Dans un tel contexte, il est compréhensible que les études québécoises sur l'insertion professionnelle se soient préoccupées surtout des jeunes diplômés des autres ordres d'enseignement. Cependant, la conclusion que les études supérieures fournissent un avantage sur le marché de l'emploi mérite d'être grandement nuancée, comme nous le verrons dans ce qui suit. Le but de cet article est de faire valoir qu'il n'est pas possible de discuter de l'insertion professionnelle des diplômées et des diplômés universitaires comme si ces derniers formaient un groupe social homogène. Une analyse des données disponibles sur leur situation sur le marché du travail deux ans après la sortie des études permettra de constater qu'il existe des distinctions importantes à l'intérieur d'une même cohorte de personnes diplômées.

\section{Pertinence historique et théorique de l'étude de l'insertion professionnelle des diplômées et des diplômés universitaires}

L'étude de la transition professionnelle et du succès sur le marché de l'emploi des diplômées et des diplômés universitaires québécois s'avère maintenant impérative, et ce, pour des raisons historiques. Dans le cadre de la réforme du système d'enseignement québécois durant les années soixante, l'université s'est vu confier une mission comprenant deux volets. Le premier volet était d'ordre économique; il s'agissait de développer une main-d'œuvre hautement qualifiée pour les nouveaux besoins du Québec moderne et pour assurer sa compétitivité sur les plans national et international. Le deuxième volet était d'ordre sociopolitique : l'université devait ouvrir ses portes à tout individu qui possédait les talents nécessaires et permettre ainsi la mobilité sociale ascendante (Commission royale d'enquête, 1963). Ces deux volets étaient intimement liés. Le Québec expérimentait alors une révolution qui le faisait passer à l'âge moderne, d'où la nécessité de développer une main-d'œuvre hautement qualifiée, que les «vieilles élites» de l'époque ne pouvaient plus fournir. L'université devait créer une nouvelle «élite» à l'aide de clientèles étudiantes provenant en grande partie de la «moyenne et petite bourgeoisie». Il en a résulté un développement fulgurant des institutions, de même 
qu'un accroissement soudain et continu de la clientèle et du nombre de diplômes décernés (Dandurand, Fournier et Bernier, 1980; LaHaye, 1990).

La plupart des systèmes d'enseignement supérieur des pays industrialisés se sont vu confier des mandats similaires avec l'apparition de réformes inspirées par l'idéologie libérale (Husén, 1989). On remet maintenant en question, un peu partout dans ces mêmes pays, la capacité des universités à remplir ces mandats (Fourastié, 1972; Sanyal, 1987; Husén, 1989) et le Québec ne saurait échapper à ce questionnement. L'université québécoise remplit-elle toujours la mission qu'elle a reçue lors de la Réforme? Cette mission est-elle toujours pertinente? Sinon, que peut-elle faire maintenant?

Ainsi, la présente crise de l'emploi a conduit à de nombreuses interrogations sur l'adéquation entre l'offre et de la demande des diplômées et des diplômés universitaires. Lors de la Réforme, on a cru au Québec, comme ailleurs dans le monde, que le développement d'une maind'œuvre hautement qualifiée favoriserait, par voie de conséquence, la croissance économique de la nation. De plus, on prévoyait à l'époque que les compétences exigées par la majorité des emplois augmenteraient constamment (Berg, 1970; Bertram, 1975; Clark, 1962; Fulton, 1984). Les pays industrialisés ont alors investi massivement dans leur système d'enseignement supérieur, voyant dans l'augmentation de la qualité de leur main-d'œuvre, un enjeu majeur de leur développement (Coombs, 1985). La diminution ou l'arrêt de la croissance économique semble maintenant remettre en question la pertinence de ces prévisions (Coombs, 1985). Des études américaines ont cherché, non sans succès, à faire valoir qu'il existe maintenant un surcroît de diplômées et de diplômés universitaires pour le nombre de postes qualifiés disponibles (Berg, 1970; Rumberger, 1981). De même, dans le cas du Québec, une étude tend à démontrer que le rythme de l'augmentation des compétences exigées sur le marché de l'emploi est plus lent que celui de l'augmentation des qualifications scolaires de la main-d'œuvre (Renaud et al., 1980; Bertcherman et al., 1991). Plusieurs auteurs observent une polarisation des compétences sur le marché, particulièrement dans le secteur tertiaire (Bertcherman et al., 1991; Fréchet, 1990), secteur qui recrute, au Québec, le plus grand nombre de diplômées et de diplômés univer- 
sitaires (Audet, 1991). Toutes ces études suscitent un questionnement sur l'adéquation entre, d'une part, les compétences exigées par les emplois disponibles et, d'autre part, les qualifications acquises par le biais de la formation universitaire. Est-il possible que plusieurs de nos diplômées et de nos diplômés se retrouvent dans des emplois dont le niveau de qualification ne correspond pas clairement à leur niveau de formation?

Il est facile d'appréhender alors l'impact potentiel de cette inadéquation sur la capacité de l'université de remplir le second volet de sa mission. Le discours libéral a eu pour effet de libérer les aspirations sociales de mobilité chez certaines familles québécoises. Les jeunes ont été soumis, d'une part, à des aspirations parentales élevées (Bernier, 1986) et, d'autre part, à la constatation que le marché du travail exigeait d'eux toujours plus de qualifications scolaires (Laperrière, 1982; Dore, 1975; Coleman et Husén, 1985). Il s'ensuit une augmentation constante de la demande d'éducation de niveau supérieur, qui résulte de l'espoir que le diplôme obtenu permettra d'améliorer le sort professionnel (Gaskell, 1991). Or, si ces jeunes se retouvent en grande partie dans des emplois déqualifiés, un désappointement envers le système d'enseignement supérieur pourrait en résulter.

Le sous-emploi des diplômées et des diplômés ne s'exprime pas seulement de façon qualitative, par la distance entre la qualification nécessaire à l'emploi et la qualification scolaire, mais également de façon quantitative, c'est-à-dire par le faible degré d'utilisation sociale des ressources qu'ils représentent (Sanyal, 1987). Ainsi qu'on pourra le constater dans ce qui suit, c'est surtout à ce niveau que les différences au sein de la cohorte de diplômées et de diplômés sont frappantes. Alors que certaines diplômées et certains diplômés sont pleinement employés, c'est-àdire qu'ils jouissent d'emploi régulier à temps plein, d'autres ne possèdent pas ce même avantage. Un bassin important de compétences est ainsi négligé ou peu exploité.

On perçoit aisément tout ce que cette situation a de dramatique; il s'agit vraiment, dans les mots d'un auteur québécois, d'une «rupture de contrat» social (Bernier, 1986). On pouvait malheureusement prévoir, au fur et à mesure que s'allongeait la crise de l'emploi, qu'un tel phéno- 
mène devait se produire. En effet, les travaux de Ledrut (1966) avaient permis de conclure que, durant une période de prospérité économique, le chômage tend à affecter surtout un certain type de travailleurs; les femmes plus que les hommes, les jeunes et les personnes âgées plus que les individus d'âge moyen et, surtout, pour les propos qui nous préoccupent, les personnes peu qualifiées (sur la base de leur scolarité) plus que celles qui possèdent de hautes qualifications. En période de récession et de crise de l'emploi cependant, ces disparités auront tendance à se niveler. Puisque la crise de l'emploi s'est déjà étendue sur presque deux décennies, on peut s'attendre à une diminution de la différence entre le taux de chômage des jeunes diplômées et diplômés universitaires, normalement moins soumis au chômage, et celui des jeunes diplômés des autres niveaux, et de l'ensemble des jeunes sur le marché du travail. Dans l'étude déjà mentionnée, l'analyse de certaines données de l'Enquête sur l'activité par Gauthier (1991) semble déjà venir appuyer cette thèse. Elle révèle une tendance au nivellement de la différence entre les taux de chômage des diplômées et des diplômés universitaires et celui des autres groupes de jeunes.

Dans un tel contexte, la probabilité de mobilité ascendante, au sein d'une même cohorte de diplômées et de diplômés universitaires, ne peut qu'être remise en question. En effet, il devient peu probable que les jeunes puissent "gravir les échelons» s'ils ne peuvent, au départ, accéder à des emplois qualifiés et à caractère typique. Un tel constat conduirait à modifier l'accent de la recherche sur le problème de l'égalité d'accès à l'enseignement supérieur, en faveur d'études sur le problème de l'utilité ou des résultats de cette formation, c'est-à-dire sur l'impact de l'enseignement supérieur sur la position sociale et économique relative des divers groupes d'individus (Coleman, 1973) ${ }^{1}$. Au Québec, toutes les études se sont clairement adressées au problème de l'égalité de l'accès (Anisef et al., 1989). Cette étude-ci permettra de commencer à considérer l'égalité des résultats, soit la capacité de la société et du marché de l'emploi québécois de permettre aux jeunes diplômés des divers programmes universitaires l'accès aux types d'emploi traditionnellement

1 Cet auteur définit l'égalité des résultats comme étant une distribution équitable des revenus. Le sens donné à cette expression ici est un peu plus large et englobe l'équité dans l'accès au travail rémunéré à caractère typique et qui permet l'utilisation de ses qualifications. 
associés à leur formation et, en fait, dans la conjoncture actuelle, à l'emploi tout court.

2. Analyse, en 1989, des résultats de la relance des diplômés du 1er cycle de 1987

De façon succincte, la recherche sur l'enseignement supérieur québécois est confrontée aux questions suivantes.

1) Les diplômées et les diplômés sont-ils quantitativement sousemployés? Sont-ils nombreux ou peu à occuper des emplois typiques, c'est-à-dire des emplois à temps plein régulier et dont la durée n'est pas déterminée au préalable?

2) Les diplômées et les diplômés sont-ils qualitativement sousemployés? Sont-ils nombreux ou peu à détenir un emploi qu'ils perçoivent être relié à leur domaine d'études, cette perception étant un indice de l'utilisation des qualifications acquises lors de la formation?

3) Peut-on établir des différences entre divers groupes de diplômées et de diplômés?

Une analyse des données d'une relance récente du ministère de l'Enseignement supérieur et de la Science devrait nous permettre de répondre à ces questions. Cette relance intitulée $Q u$ 'advient-il des diplômées et des diplômés universitaires? (Audet, 1991) a rejoint 23621 personnes diplômées du premier cycle universitaire. Cette enquête avait pour but de décrire, deux ans après la sortie des études, la situation sur le marché de l'emploi de l'ensemble des diplômés du premier cycle de 1987 des universités québécoises. Les catégories suivantes ont été utilisées pour la présentation des résultats.

Diplômé et diplômée en emploi. - Toute personne diplômée qui avait un emploi rémunéré et qui travaillait soit à temps plein, soit à temps partiel. Les personnes qui cumulent études et travail à temps plein font partie de cette catégorie.

Diplômé et diplômée sans emploi. - Toute personne diplômée sans emploi rémunéré et qui cherchait un emploi. 
Diplômé et diplômée aux études. - Toute personne diplômée sans emploi et qui étudiait, ou qui avait un emploi à temps partiel tout en étudiant comme occupation principale.

Diplômé inactif ou diplômée inactive. - Toute personne diplômée qui rencontrait toutes les conditions suivantes : ne pas avoir d'emploi rémunéré, ne pas être aux études, ne pas avoir occupé un emploi et ne pas en chercher.

La catégorie «diplômé et diplômée en emploi» se divise en souscatégories.

Diplômé et diplômée en emploi à temps plein. - Toute personne diplômée qui a un emploi de trente heures ou plus par semaine. Une personne diplômée travaille donc à temps partiel si elle travaille moins de trente heures par semaine.

Diplômé et diplômée en emploi relié aux études. - Toute personne diplômée qui a un emploi qu'elle perçoit comme correspondant positivement au domaine d'études de son diplôme. Cette catégorie nous servira d'indice de l'utilisation des compétences acquises lors de la formation.

Diplômé et diplômée en emploi régulier. - Toute personne diplômée qui a un emploi qui ne comportait à l'embauche aucune limitation quant à sa durée. Dans le cas inverse, la personne diplômée sera dite en emploi temporaire.

\subsection{Les résultats globaux de la relance}

Les résultats globaux pertinents pour notre étude, tels qu'ils apparaissent dans le rapport d'enquête, sont reproduits au tableau 1. 
Tableau 1 -Situation, en 1989, sur le marché de l'emploi des personnes diplômées des universités québécoises en 1987

\begin{tabular}{|l|c|}
\hline Catégories & Résultats \\
\hline Nombre total de diplômés et de diplômées & 23621 \\
(dont on soustrait) & $13,2 \%$ \\
Diplômés et diplômées aux études & $1,8 \%$ \\
Diplômés inactifs et diplômées inactives & 20101 \\
= Nombre de diplômés et de diplômées disponibles à l'emploi & $8,1 \%$ \\
(dont on soustrait) & 18472 \\
Diplômés et diplômées sans emploi & \\
Nombre de diplômés occupés et de diplômées occupées & $90,2 \%$ \\
À temps plein & $72,7 \%$ \\
- relié aux études & $17,5 \%$ \\
- non relié aux études & $71,8 \%$ \\
- tégulier & $18,4 \%$ \\
À temporaire & $9,8 \%$ \\
- relié au domaine d'études & $6,8 \%$ \\
- non relié au domaine d'études & $3,0 \%$ \\
- régulier & $3,9 \%$ \\
\hline
\end{tabular}

Source. - Audet, 1991

La présentation du tableau peut paraître insolite au premier regard. Elle est rendue nécessaire pour comprendre la façon dont l'auteur du rapport d'enquête effectue ses calculs. Ce dernier s'est servi des définitions des statistiques de l'emploi pour parvenir aux résultats présentés. Ainsi, le taux de chômage des personnes diplômées n'est pas calculé sur le nombre total de ces dernières mais seulement sur la portion de l'échantillon qui est disponible à l'emploi. On a donc calculé la proportion, sur le nombre total de personnes diplômées $(\mathrm{N}=23621)$, de celles qui sont demeurées aux études et de celles qui se déclarent inactives. Par définition, dans les statistiques de l'emploi, ces deux groupes sont considérés comme ne faisant pas partie de la population disponible à l'emploi. On a ensuite soustrait ces deux catégories du nombre total de personnes diplômées pour obtenir le nombre réel de celles qui sont disponibles pour l'emploi $(\mathrm{N}=20101)$. Il s'ensuit que la statistique suivante, le pourcentage de personnes diplômées sans emploi (ou le taux de chômage à $8,1 \%$ ), conformément à la définition utilisée, est calculée 
sur le nombre de personnes diplômées disponibles et non pas sur le nombre total de personnes diplômées.

L'auteur du rapport d'enquête a, par la suite, soustrait le nombre de personnes diplômées en chômage du nombre de personnes diplômées disponibles pour obtenir le nombre de personnes diplômées occupées $(\mathrm{N}=18472$ ). Les statistiques qui suivent sont toutes calculées sur la base de ce nouveau nombre. Ainsi, le pourcentage de personnes diplômées en emploi à temps plein est de 90,2\% du nombre de personnes diplômées occupées, et non pas de l'ensemble des personnes diplômées. Une proportion importante de l'échantillon $(\mathrm{N}=5149)$ a été ainsi perdue en cours de route. Il en est de même pour les statistiques qui suivent celle-ci jusqu'à la fin du tableau 1.

Cette façon de calculer les divers pourcentages est conforme aux définitions des indicateurs du marché du travail (population active, taux de chômage, emploi à temps plein et partiel). Bien qu'il s'agisse là de la façon la plus courante de présenter les statistiques sur l'emploi, la pertinence de ces indicateurs pour les propos de la prise de décision en éducation peut être remise en question. Le fait de soustraire ainsi de l'échantillon certains groupes d'individus peut conduire à une sous-évaluation des difficultés d'accès aux premiers emplois des diplômées et des diplômés universitaires. Cette sous-évaluation pourrait alors conduire à des décisions peu judicieuses de la part des étudiantes et des étudiants lors de la sélection d'un programme d'études et de la part des administrateurs, lors de l'élaboration de plans de développement des programmes de formation.

$\mathrm{Au}$ lieu des indicateurs classiques qui n'en conservent pas moins leur pertinence, des économistes britanniques (Taylor, 1986a; Taylor 1986b; Taylor et Johnes, 1989) ont suggéré que les calculs de proportion devraient plutôt être basés sur l'ensemble d'une cohorte de diplômés, ce qui permet ainsi d'apprécier le succès des jeunes à obtenir (ou, comme ce sera exprimé par la suite, le pouvoir des diplômes à rendre accessible) un ou des premiers emplois. Pour pouvoir calculer de tels indicateurs, il a fallu d'abord transformer les résultats bruts de la relance, de façon à décrire la distribution de l'ensemble des diplômés dans chacune des catégories. On parvient alors aux résultats du tableau 2. 
Dans ce tableau, les pourcentages des quatre premières catégories, mutuellement exclusives, s'additionnent de façon à représenter l'ensemble des personnes diplômées (total $=100 \%$ ). Le total des pourcentages des sous-catégories «diplômés et diplômées en emploi temps plein» et «diplômés et diplômées en emploi à temps partiel» correspond également au pourcentage de la catégorie «diplômés et diplômées en emploi» $(78,2 \%)$. On notera que les deux autres sous-catégories ont été croisées avec la première. Les résultats permettent de constater la répartition de l'ensemble des personnes diplômées.

Tableau 2 - Distribution de l'ensemble des personnes diplômées des universités québécoises en 1987 par catégories d'emploi en 1989

\begin{tabular}{|l|c|}
\hline Catégories & Résultats \\
\hline Nombre total de diplômés et de diplômées & 23621 \\
Diplômés et diplômées aux études & $13,2 \%$ \\
Diplômés inactifs et diplômées inactives & $1,8 \%$ \\
Diplômés et diplômées sans emploi & $6,8 \%$ \\
Diplômés et diplômées en emploi & $78,2 \%$ \\
- diplômés et diplômées en emploi à temps plein & $70,6 \%$ \\
- relié aux études & $56,8 \%$ \\
non relié aux études & $13,7 \%$ \\
- régulier & $56,2 \%$ \\
- temporaire & $14,3 \%$ \\
- diplômés et diplômées en emploi à demps partiel & $7,6 \%$ \\
- non relié au domaine d'études & $5,3 \%$ \\
- régulier & $2,3 \%$ \\
— temporaire & $3,0 \%$ \\
\hline
\end{tabular}

Source. - Audet, 1991 et calcul de l'auteur

Cette présentation des statistiques conduit à des observations fort différentes de celles qui découlent de la première. On se rend compte qu'à peine trois quarts de l'ensemble des personnes diplômées occupent effectivement un emploi quelconque, et à peine un peu plus de la moitié d'entre elles ont un emploi permanent $(56,2 \%$ au lieu de $71,8 \%)$ ou un emploi relié au domaine d'études $(56,8 \%$ au lieu de $72,7 \%)$, et ce, deux ans après leur sortie de l'université. 
Bien qu'il soit nécessaire de considérer ces résultats globaux, cela ne nous apprend encore que peu de choses. Quelles sont les différences entre les diplômées et les diplômés des programmes différents? Y a-t-il des différences également entre les sexes? Il sera possible de répondre à ces questions puisque la relance utilisée donne des résultats distincts et utilisables pour un total de quatre-vingt-treize programmes québécois. La même modification des statistiques descriptives présentées a donc été effectuée pour chacun de ces programmes. La distribution des personnes diplômées dans les diverses catégories pour le programme de bioagronomie est présentée au tableau 3, à titre illustratif.

Tableau 3 - Distribution des personnes diplômées en 1987 du programme de bio-agronomie par catégories d'emploi au Québec en 1989

\begin{tabular}{|c|c|c|c|}
\hline Catégories & Hommes & Femmes & Total \\
\hline Nombre de diplômés et diplômées & 112 & 117 & 229 \\
\hline Diplômés et diplômées aux études & $19,7 \%$ & $18,9 \%$ & $19,3 \%$ \\
\hline $\begin{array}{l}\text { Diplômés inactifs et diplômées } \\
\text { inactives }\end{array}$ & $0,0 \%$ & $4,1 \%$ & $2,1 \%$ \\
\hline Diplômés et diplômées sans emploi & - & - & $3,9 \%$ \\
\hline $\begin{array}{l}\text { Diplômés et diplômées en emploi } \\
\text { à temps plein }\end{array}$ & $76,7 \%$ & $68,6 \%$ & $72,5 \%$ \\
\hline $\begin{array}{l}\text { à temps plein régulier } \\
\text { - à temps plein temporaire } \\
\text { - à temps plein relié au domaine }\end{array}$ & $\begin{array}{l}54,0 \% \\
22,9 \% \\
69,5 \%\end{array}$ & $\begin{array}{l}44,0 \% \\
24,4 \% \\
54,9 \%\end{array}$ & $\begin{array}{l}48,9 \% \\
23,6 \% \\
62,0 \%\end{array}$ \\
\hline $\begin{array}{l}\text { Diplômés et diplômées en emploi } \\
\text { à temps partiel }\end{array}$ & $0,0 \%$ & $4,1 \%$ & $2,1 \%$ \\
\hline
\end{tabular}

Il est aisé de se rendre compte, pour ce programme comme pour l'ensemble des personnes diplômées, que les chiffres semblent décrire une toute autre réalité lorsqu' on ramène les statistiques de façon à refléter la distribution de toute une cohorte dans chaque catégorie. On passe ainsi, pour les deux sexes, d'un taux d'emploi à temps plein de $97,2 \%$ à $72,5 \%$. Cette différence est considérable. De même, l'emploi à temps plein relié au domaine d'études passe de $83,1 \%$ à $62,0 \%$, et l'emploi à temps plein régulier de $65,5 \%$ à $48,9 \%$. 
Lorsque toutes les statistiques descriptives ont été ainsi modifiées, il a été possible de calculer de nouveaux indicateurs, sous forme de ratios, qui permettent de décrire la proportion des personnes diplômées des divers programmes ayant accédé à certains types d'emploi. En s'inspirant de ceux présentés par les économistes britanniques (Taylor et Johnes, 1989), les deux ratios suivants ont été construits.

Indice d'emploi à temps plein régulier

$$
\mathrm{IPR}=\frac{\mathrm{TPR}}{\mathrm{TPR}+\mathrm{TPT}+\mathrm{TP}+\mathrm{ET}+\mathrm{CH}}
$$

où $\quad \mathrm{TPR}=$ pourcentage de personnes diplômées en emploi à temps plein et régulier;

TPT $=$ pourcentage de personnes diplômées en emploi à temps plein et temporaire;

$\mathrm{TP}=$ pourcentage des personnes diplômées en emploi à temps partiel;

$\mathrm{ET}=$ pourcentage des personnes diplômées dont la principale occupation était d'être aux études lors de l'enquête;

$\mathrm{CH}=$ pourcentage des personnes diplômées sans emploi.

Indice d'emploi à temps plein relié au domaine d'études

$$
\mathrm{IPL}=\frac{\mathrm{TPL}}{\mathrm{TPR}+\mathrm{TPT}+\mathrm{TP}+\mathrm{ET}+\mathrm{CH}}
$$

où TPL $=$ le pourcentage de personnes diplômées en emploi à temps plein qui jugent que cet emploi est relié à leur domaine d'études.

Ces ratios sont donc respectivement un indicateur du pouvoir fourni par un diplôme à la personne diplômée d'accéder à un emploi typique, selon la définition opératoire qui en a été faite précédemment, et un indicateur du pouvoir fourni par le diplôme à la personne diplômée d'accéder à un emploi relié à son domaine d'études. Le tableau 4 présente les résultats du calcul du premier ratio pour les personnes diplômées des quatrevingt-treize programmes différents, en ordre décroissant. Certains pro- 
grammes, retenus pour les propos de la relance, ont dû être ignorés ${ }^{2}$ à cause du faible nombre de leurs personnes diplômées et de plusieurs données manquantes.

L'aspect le plus frappant est l'étendue considérable des résultats. Le programme au ratio le plus élevé, actuariat, est séparé par soixantedix-neuf points de pourcentage de celui dont le ratio est le moins élevé, philosophie. La distribution est très progressive et s'approche d'une courbe normale. Les programmes ont ensuite été regroupés par famille et par type de formation, professionnelle ou non professionnelle (tableau 5). Cette dernière distinction est basée sur la définition suivante : un programme universitaire «est considéré professionnel lorsqu'il est possible de lui associer une occupation précise, qui implique l'application de savoir-faire distincts, et qui peut être liée à un secteur particulier de la production de biens ou de services» (Howes et Howes, 1982; Loose, $1985)^{3}$. Tous les programmes qui se situent au-dessus d'un écart-type $(=0,22)$ de la moyenne $(=0,54)$ sont des programmes de formation à caractère professionnel. Tous sont également soit des programmes des sciences de l'administration (finance, administration des affaires, comptabilité), des sciences appliquées (actuariat, génie mécanique, génie informatique) ou des sciences de la santé (médecine dentaire, optométrie).

2 Ces programmes sont les suivants : technologie de la production automatisée, technologie de la construction civile, géographie physique, économie des affaires, méthodes quantitatives et recherches opérationnelles, management des richesses naturelles, administration scolaire, production, météorologie, études allemandes, études anciennes, arts et traditions populaires, ressources humaines, bibliothéconomie, études hispaniques, photographie, environnement et écologie, animation culturelle et sociale.

3 Tout programme pour lequel subsistait un doute n'a pas été inclus parmi les programmes professionnels. C'est le cas de certains programmes dont la raison d'être semble plutôt de préparer à résoudre un type de problème qu'il est possible de rencontrer dans un grand nombre de secteurs del'activité économique : relations industrielles, traduction, criminologie, maths-stats, maths-info, sciences de l'activité physique. Ces distinctions mériteraient encore d'être précisées. 
Tableau 4 - Indices, en 1989 au Québec, des emplois à temps plein régulier (IPR), pour 93 programmes de premier cycle universitaire, cohorte de 1987

\begin{tabular}{lcl} 
Programmes & Ratio & Programmes \\
Actuariat & 0,94 & Design - Communication graphique \\
Gestion des entreprises & 0,94 & Physiothérapie \\
Génie industriel & 0,94 & Design de l'environnement \\
Technologie de l'électricité & 0,91 & Criminologie \\
Comptabilité & 0,89 & Ergothérapie \\
Technologie de la construction & & Médecine \\
mécanique & 0,88 & Economique \\
Marketing & 0,87 & Mathématiques - Informatique \\
Génie informatique & 0,86 & Traduction \\
Médecine dentaire & 0,85 & Architecture de paysage \\
Génie minier & 0,85 & Géomatique \\
Optométrie & 0,85 & Orthophonie - Audiologie \\
Génie mécanique & 0,84 & Gestion des interventions \\
Génie unifié & 0,82 & touristiques \\
Génie civil, de la construction & & Sciences infirmières \\
et du transport & 0,82 & Études géopolitiques \\
Journalisme & 0,81 & Sciences des aliments \\
Information de gestion - Traitement & & Service social \\
des données & 0,78 & Génie géologique \\
Administration des affaires & 0,78 & Anglais - langue et littérature \\
Informatique & 0,77 & Agriculture - Bio-agronomie \\
Génie électrique, électronique, & & Communication \\
des communications & 0,75 & Génie rural \\
Génie chimique & 0,75 & Enseignement préscolaire \\
Médecine vétérinaire & 0,74 & et primaire \\
Relations industrielles & 0,73 & Administration hospitalière \\
Génie métallurgique & 0,73 & Théologie \\
Architecture & 0,72 & Chimie \\
Économie rurale - Agro-économie & 0,72 & Récréation \\
Pharmacie & 0,72 & Aménagement - Urbanisme - \\
Droit & 0,68 & Études urbaines \\
Enseignement professionnel & & Psychoéducation - Psychopédagogie \\
et technique & 0,67 & Foresterie \\
Design industriel & 0,66 & Enseignement en adaptation \\
Mathématiques - Statistiques & 0,66 & scolaire \\
& & \\
\hline
\end{tabular}

\begin{tabular}{cl} 
Ratio & Programmes \\
0,65 & Génie physique \\
0,65 & Linguistique \\
0,64 & Géologie \\
0,63 & Sciences politiques \\
0,63 & Économie de la consommation \\
0,62 & Sexologie \\
0,61 & Géographie \\
0,61 & Enseignement spécialisé (discipline) \\
0,60 & Français - langue et littérature \\
0,60 & Éducation physique \\
0,60 & Sociologie \\
0,59 & Diététique - Nutrition \\
& Information scolaire \\
0,58 & et professionnelle \\
0,54 & Beaux-arts - Arts plastiques \\
0,54 & Danse \\
0,54 & Art dramatique \\
0,53 & Sciences de l'activité physique \\
0,52 & Biologie \\
0,52 & Histoire \\
0,50 & Psychologie \\
0,49 & Biochimie \\
0,47 & Cinéma \\
& Microbiologie et sciences \\
0,46 & fondamentales en médecine \\
0,45 & Orientation \\
0,44 & Design appliqué \\
0,44 & Anthropologie \\
0,43 & Physique \\
& Histoire de l'art \\
0,43 & Musicologie \\
0,43 & Musique \\
0,43 & Philosophie \\
0,42 & \\
\hline & \\
\hline
\end{tabular}


Tableau 5 - Une classification des programmes par famille et par type de formation ${ }^{4}$

Sciences
de l'adminis tration
Administration des affaires
(P)
Comptabilité (P)
Finance (P)
Gestion des entreprises $(\mathrm{P})$
Gestion des interventions
touristiques $(\mathrm{P})$
Marketing (P)
Information de gestion (P)
Relations industrielles

Arts ef beaux-arts

Art dramatique

Beaux-arts et arts plastiques

Cinéma

Danse

Design appliqué $(\mathrm{P})$

Communication graphique (P)

Histoire de l'art

Musique

Musicologie

\section{Langues \\ et littérature \\ Anglais \\ Français-langue et \\ littérature \\ Linguistique \\ Traduction}

Sciences pures

Mathématiques et

statistiques

Physique

Biochimie

Biologie

Microbiologie

Géologie

Chimie

Sciences appliquées

Génie chimique $(\mathrm{P})$

Génie civil (P)
Génie électrique (P)

Génie géologique $(\mathbb{P})$

Génie industriel (P)

Génie informatique $(\mathrm{P})$

Génie mécanique $(\mathrm{P})$

Génie métallurgique $(\mathrm{P})$

Génie minier $(\mathbb{P})$

Génie physique $(P)$

Génie rural $(\mathrm{P})$

Génie unifié $(P)$

Géomatique (P)

Foresterie (P)

Technologie de l'électricité (P)

Technologie de la construction mécanique (P)

Actuariat $(\mathrm{P})$

Informatique $(\mathrm{P})$

Maths et informatique

Sciences sociales

et humaines

Droit (P)

Communication

Histoire

Journalisme (P)

Sociologie

Philosophie

Théologie

Anthropologie

Criminologie

Économique

Études géopolitiques

Géographie

Psychologie 5

Sciences politiques

Service social $(\mathrm{P})$

\section{Sciences de la santé}

Sexologie (P)

Ergothérapie $(\mathrm{P})$

Orthophonie et audiologie (P)

Physiothérapie (P)

Médecine (P)

Médecine dentaire $(\mathrm{P})$
Administration hospitalière $(\mathbb{P})$

Optométrie $(\mathrm{P})$

Sciences infirmières $(\mathrm{P})$

Pharmacie (P)

Sciences de l'activité physique

Sciences

de l'agriculture

et de l'alimentation

Économie de la

consommation

Diététique et nutrition (P)

Agriculture ebio-agronomie (P)

Économie rurale et agroéconomique (P)

Médecine vétérinaire (P) Science et technologie des aliments $(\mathbb{P})$

Urbanisme et design

Aménagement/Urbanisme/ Etudes urbaines (P)

Architecture (P)

Architecture de paysage $(\mathrm{P})$

Design de l'environnement (P)

Design industriel $(\mathrm{P})$

Éducation

et domaines connexes

Enseignement préscolaire et primaire $(P)$

Enseignement spécialisé (discipline) $(\mathrm{P})$

Enseignement en adaptation scolaire (P)

Psychoéducation/Psycho-

pédagogie $(\mathrm{P})$

Enseignement

professionnel et technique (P)

Education physique (P)

Information scolaire et professionnelle (P)

Orientation $(\mathrm{P})$

Récréation $(\mathrm{P})$

4 La mention $(\mathrm{P})$ désigne un programme à caractère professionnel.

5 Le programme de psychologie n'a pas été inclus parmi les programmes professionnels parce qu'acquérir le droit d'exercice de la profession nécessite l'obtention d'une maîtrise. 
Au contraire, tous les programmes en-dessous d'un écart-type de la moyenne sont soit des programmes de sciences sociales et humaines (psychologie, anthropologie, histoire), des programmes de beaux-arts et d'arts (cinéma, histoire de l'art, musicologie) ou des programmes de sciences pures (physique, biologie, biochimie). On ne retrouve qu'un seul programme des sciences de la santé (sciences de l'activité physique) et qu'un programme à caractère fortement professionnel (orientation) à un écart-type de distance ou plus en-dessous de la moyenne.

Pour permettre une lecture plus facile, les résultats décrits au tableau 4 ont été étalonnés de façon à pouvoir être regroupés sous six cotes, décrites dans le tableau 6. Ces cotes sont basées sur l'écart-type de la distribution des ratios. La cote 1, par exemple, regroupe les programmes dont les ratios sont supérieurs à la moyenne d'au moins un écart-type. Ces programmes sont donc ceux dont les personnes diplômées ont le plus grand pouvoir d'obtention d'un emploi à temps plein régulier, relativement à l'ensemble des diplômées et des diplômés de tous les programmes. À l'inverse, la cote 6 regroupe les programmes dont les ratios sont inférieurs à la moyenne d'au moins un écart-type et qui fournissent à leurs diplômés le pouvoir relatif le plus faible. Les programmes ont également été regroupés par famille et par type de formation (tableau 7).

Tableau 6 - Description des cotes utilisées pour regrouper les indices d'emploi à temps plein régulier

et les indices d'emploi à temps plein relié au domaine d'études

\begin{tabular}{cl}
\hline Cote & Description \\
\hline 1 & Un écart-type ou plus au-dessus de la moyenne \\
2 & D'un demi-écart-type à un écart-type au-dessus de la moyenne \\
3 & Entre la moyenne et un demi-écart-type au-dessus de la moyenne \\
4 & Entre la moyenne et un demi-écart-type au-dessous de la moyenne \\
5 & D'un demi-écart-type à un écart-type au-dessous de la moyenne \\
6 & Un écart-type ou plus au-dessous de la moyenne \\
\hline
\end{tabular}


Tableau 7 - Indices, en 1989 au Québec, du pouvoir relatif d'obtention d'un emploi régulier à temps plein selon la famille et le type de formation pour 1993, programmes universitaires de premier cycle, cohorte de 1987

\begin{tabular}{|c|c|c|c|c|c|c|c|}
\hline $\begin{array}{l}\text { F amilles } \\
\text { de formation }\end{array}$ & $\begin{array}{c}\text { Profes- } \\
\text { sionnels (N) }\end{array}$ & 1 & 2 & 3 & 4 & 5 & 6 \\
\hline $\begin{array}{l}\text { Sciences } \\
\text { de l'administration }\end{array}$ & 7 & 6 & 0 & 1 & 0 & 0 & 0 \\
\hline Arts et beaux-arts & 2 & 0 & 1 & 0 & 0 & 0 & 1 \\
\hline Langues et littérature & 0 & 0 & 0 & 0 & 0 & 0 & 0 \\
\hline Sciences pures & 0 & 0 & 0 & 0 & 0 & 0 & 0 \\
\hline Sciences appliquées & 18 & 10 & 3 & 1 & 3 & 1 & 0 \\
\hline $\begin{array}{l}\text { Sciences sociales } \\
\text { et humaines }\end{array}$ & 3 & 1 & 1 & 0 & 1 & 0 & 0 \\
\hline Sciences de la santé & 10 & 2 & 2 & 3 & 2 & 1 & 0 \\
\hline $\begin{array}{l}\text { Sciences } \\
\text { de l'agriculture }\end{array}$ & 5 & 0 & 2 & 0 & 2 & 1 & 0 \\
\hline Urbanisme et design & 5 & 0 & 2 & 2 & 1 & 0 & 0 \\
\hline $\begin{array}{l}\text { Éducation et domaines } \\
\text { connexes }\end{array}$ & 9 & 0 & 1 & 0 & 3 & 3 & 2 \\
\hline Totaux & 59 & 19 & 12 & 7 & 12 & 6 & 3 \\
\hline $\begin{array}{l}\text { Familles } \\
\text { de formation }\end{array}$ & $\begin{array}{l}\text { Non profes- } \\
\text { sionnels (N) }\end{array}$ & 1 & 2 & 3 & 4 & 5 & 6 \\
\hline $\begin{array}{l}\text { Sciences } \\
\text { de l'administration }\end{array}$ & 1 & 0 & 1 & 0 & 0 & 0 & 0 \\
\hline Arts et beaux-arts & 7 & 0 & 0 & 0 & 0 & 0 & 7 \\
\hline Langues et littérature & 4 & 0 & 0 & 1 & 1 & 2 & 0 \\
\hline Sciences pures & 7 & 0 & 1 & 0 & 1 & 1 & 4 \\
\hline Sciences appliquées & 1 & 0 & 0 & 1. & 0 & 0 & 0 \\
\hline $\begin{array}{l}\text { Sciences sociales } \\
\text { et humaines }\end{array}$ & 12 & 0 & 0 & 2 & 3 & 3 & 4 \\
\hline Sciences de la santé & 1 & 0 & 0 & 0 & 0 & 0 & 1 \\
\hline $\begin{array}{l}\text { Sciences } \\
\text { de l'agriculture }\end{array}$ & 1 & 0 & 0 & 0 & 0 & 1 & 0 \\
\hline Urbanisme et design & 0 & 0 & 0 & 0 & 0 & 0 & 0 \\
\hline $\begin{array}{l}\text { Éducation et domaines } \\
\text { connexes }\end{array}$ & 0 & 0 & 0 & 0 & 0 & 0 & 0 \\
\hline Totaux & 34 & $\mathbf{0}$ & 2 & 4 & 5 & 7 & 16 \\
\hline Grand total & 93 & & & & & & \\
\hline
\end{tabular}


Tableau 8 - Indices, en 1989 au Québec, des emplois à temps plein relié au domaine d'études (IPL), pour 93 programmes de premier cycle universitaire, cohorte de 1987

Programmes
Optométrie
Médecine dentaire
Médecine
Génie unifié
Génie minier
Comptabilité
Informatique
Actuariat
Géomatique
Génie civil, de la construction
et du transport
Architecture
Foresterie
Génie industriel
Pharmacie
Physiothérapie
Génie mécanique
Génie informatique
Ergothérapie
Technologie de la construction
mécanique
Économie rurale - Agro-économie
Gestion des entreprises
Technologie de l'électricité
Droit
Information de gestion - Traitement
des données
Génie chimique
Génie électrique, électronique,
des communications
Architecture du paysage
Mathématiques - Informatique
Médecine vétérinaire
Orthophonie - Audiologie
Service social

\begin{tabular}{|c|c|c|c|}
\hline Ratio & Programmes & Ratio & Programmes \\
\hline 0,96 & Enseignement professionnel & & Récréation \\
\hline 0,95 & et technique & 0,73 & Diététique - Nutrition \\
\hline 0,92 & Administration des affaires & 0,73 & Éducation physique \\
\hline 0,91 & Design - Communication graphique & 0,72 & Traduction \\
\hline 0,90 & Administration hospitalière & 0,72 & Art dramatique \\
\hline 0,90 & Marketing & 0,71 & Théologie \\
\hline 0,90 & Génie gêologique & 0,70 & Économique \\
\hline 0,88 & Criminologie & 0,70 & Cinéma \\
\hline \multirow[t]{2}{*}{0,88} & Sciences infirmières & 0,70 & Danse \\
\hline & Finance & 0,68 & Beaux-arts - Arts plastiques \\
\hline 0,86 & Génie métallurgique & 0,66 & Français - langue et littérature \\
\hline 0,84 & Journalisme & 0,66 & Sciences de l'activité physique \\
\hline 0,84 & Agriculture - Bio-agronomie & 0,63 & Anglais - langue et litterature \\
\hline 0,83 & Enseignement en adaptation scolaire & 0,62 & Biologie \\
\hline 0,83 & Sciences des aliments & 0,61 & Microbiologie et sciences \\
\hline 0,83 & Psychoéducation - Psychopédagogie & 0,60 & fondamentales en médecine \\
\hline 0,82 & Design de l'environnement & 0,60 & Sociologie \\
\hline 0,82 & Génie rural & 0,59 & Biochimie \\
\hline \multirow[t]{2}{*}{0,82} & Enseignement préscolaire et primaire & 0,58 & Géographie \\
\hline & Design appliqué & 0,57 & Psychologie \\
\hline 0,82 & Orientation & 0,55 & Musique \\
\hline 0,82 & Relations industrielles & 0,55 & Études géopolitiques \\
\hline 0,82 & Communication & 0,53 & Physique \\
\hline 0,81 & Chimie & 0,52 & Sciences politiques \\
\hline \multirow[t]{2}{*}{0,81} & Mathématiques - Statistiques & 0,51 & Linguistique \\
\hline & Gestion des interventions & & Musicologie \\
\hline 0,80 & touristiques & 0,50 & Sexologie \\
\hline \multirow[t]{2}{*}{0,79} & Design industriel & 0,49 & Anthropologie \\
\hline & Enseignement spécialisé (discipline) & 0,48 & Économie de la consommation \\
\hline 0,79 & Information scolaire & & Histoire \\
\hline 0,78 & et professionnelle & 0,48 & Histoire de l'art \\
\hline 0,77 & Génie physique & 0,46 & Philosophie \\
\hline 0,76 & Géologie & 0,46 & \\
\hline 0,75 & Aménagement - Urbanisme - & & \\
\hline 0,75 & Études urbaines & 0,45 & \\
\hline
\end{tabular}


Le faible pouvoir des diplômés des programmes non professionnels à obtenir un emploi typique apparaît clairement. Presque la moitié de ces programmes sont regroupés sous la cote 6 , et on n'en retrouve que deux dans la cote 1 et 2 . Au contraire, plus de la moitié des programmes à caractère professionnel (31/59) sont regroupés sous ces cotes. Le pouvoir d'obtention d'un emploi «typique» des personnes diplômées de ces programmes est donc beaucoup plus grand.

Ce qui vient d'être dit semble beaucoup plus juste pour certains types de formation à caractère professionnel que d'autres. Ainsi, le plus grand pouvoir est détenu par les personnes diplômées de programmes des sciences de l'administration ( $6 / 8$ dans la première cote) et des sciences appliquées (13/18 dans les deux premières cotes).

Le tableau 8 présente les résultats du calcul des indices d'emploi à temps plein relié au domaine d'études. On observe des résultats assez similaires à ceux obtenus par le calcul de l'indice précédent. L'étendue est encore considérable, allant d'un indice de 0,96 (optométrie) à 0,04 (philosophie), pour une différence de 0,92. La distribution suit une courbe à peu près normale. De nouveau, tous les programmes à un écarttype $(=0,25)$ ou plus au-dessus de la moyenne $(=0,56)$ sont des programmes à caractère professionnel. Quant à la famille de formation, ces programmes sont soit des programmes des sciences appliquées (géomatique, génie civil, génie informatique), des programmes des sciences de l'administration (comptabilité, gestion des entreprises) et on retrouve également plusieurs programmes des sciences de la santé (optométrie, ergothérapie, pharmacie, physiothérapie), un programme d'urbanisme et de design (architecture) et un programme des sciences de l'agriculture et de l'alimentation (économie rurale et agro-économie). Les origines de programme, selon la famille de formation, des personnes diplômées qui ont le plus de pouvoir d'obtention d'un emploi relié à leur domaine d'études sont donc plus variées que celles des personnes ayant le plus de pouvoir d'obtenir un emploi à temps plein régulier. Quant aux personnes diplômées qui possèdent le plus faible pouvoir d'obtention d'un emploi relié à leur domaine d'études, elles proviennent de programmes d'arts et de beaux-arts (arts plastiques, musicologie, histoire de l'art), de programmes des sciences sociales et humaines (philosophie, géographie, sciences politiques), de programmes des sciences pures (biochimie, biologie, physique), de programmes de langues et de littérature (français, 
linguistique) et on compte un programme des sciences de l'agriculture et de l'alimentation (économie de la consommation).

Tableau 9 - Indices, en 1989 au Québec, du pouvoir relatif d'obtention d'un emploi régulier relié au domaine d'études selon la famille et le type de formation pour 1993, programmes universitaires de premier cycle, cohorte de 1987

\begin{tabular}{|c|c|c|c|c|c|c|c|}
\hline $\begin{array}{l}\text { Familles } \\
\text { de formation }\end{array}$ & $\begin{array}{c}\text { Profes- } \\
\text { sionnels (N) }\end{array}$ & 1 & 2 & 3 & 4 & 5 & 6 \\
\hline $\begin{array}{l}\text { Sciences } \\
\text { de l'administration }\end{array}$ & 7 & 2 & 4 & 0 & 1 & 0 & 0 \\
\hline Arts et beaux-arts & 2 & 0 & 1 & 1 & 0 & 0 & 0 \\
\hline Langues et littérature & 0 & 0 & 0 & 0 & 0 & 0 & 0 \\
\hline Sciences pures & 0 & 0 & 0 & 0 & 0 & 0 & 0 \\
\hline Sciences appliquées & 18 & 11 & 4 & 2 & 1 & 0 & 0 \\
\hline $\begin{array}{l}\text { Sciences sociales } \\
\text { et humaines }\end{array}$ & 3 & 0 & 2 & 1 & 0 & 0 & 0 \\
\hline Sciences de la santé & 10 & 6 & 3 & 0 & 0 & 0 & 1 \\
\hline $\begin{array}{l}\text { Sciences } \\
\text { de l'agriculture }\end{array}$ & 5 & 1 & 1 & 2 & 1 & 0 & 0 \\
\hline Urbanisme et design & 5 & 1 & 1 & 1 & 2 & 0 & 0 \\
\hline $\begin{array}{l}\text { Éducation et domaines } \\
\text { connexes }\end{array}$ & 9 & 0 & 1 & 3 & 5 & 0 & 0 \\
\hline Totaux & 59 & 21 & 17 & 10 & 10 & 0 & 1 \\
\hline $\begin{array}{l}\text { Familles } \\
\text { de formation }\end{array}$ & $\begin{array}{l}\text { Non profes- } \\
\text { sionnels }(\mathrm{N})\end{array}$ & 1 & 2 & 3 & 4 & 5 & 6 \\
\hline $\begin{array}{l}\text { Sciences } \\
\text { de l'administration }\end{array}$ & 1 & 0 & 0 & 0 & 0 & 0 & 0 \\
\hline Arts et beaux-arts & 7 & 0 & 0 & 0 & 1 & 3 & 4 \\
\hline Langues et littérature & 4 & 0 & 0 & 0 & 0 & 1 & 3 \\
\hline Sciences pures & 7 & 0 & 0 & 0 & 3 & 0 & 4 \\
\hline Sciences appliquées & 1 & 0 & 0 & 0 & 0 & 0 & 0 \\
\hline $\begin{array}{l}\text { Sciences sociales } \\
\text { et humaines }\end{array}$ & 12 & 0 & 1 & 0 & 1 & 2 & 8 \\
\hline Sciences de la santé & 1 & 0 & 1 & 0 & 0 & 0 & 1 \\
\hline $\begin{array}{l}\text { Sciences } \\
\text { de l'agriculture }\end{array}$ & 1 & 0 & 0 & 0 & 0 & 0 & 1 \\
\hline Urbanisme et design & 0 & 0 & 0 & 0 & 0 & 0 & 0 \\
\hline $\begin{array}{l}\text { Éducation et domaines } \\
\text { connexes }\end{array}$ & 0 & 0 & 0 & 0 & 0 & 0 & 0 \\
\hline Totaux & 34 & 0 & 2 & 0 & 5 & 6 & 21 \\
\hline
\end{tabular}


Pour cet indice comme pour le précédent, les ratios ont été regroupés par cotes (tableau 9), qui deviennent alors des indices relatifs du pouvoir des personnes diplômées d'obtenir un emploi relié à leur domaine d'études. La lecture du tableau 9 conduit encore à des conclusions semblables à celles découlant de la lecture du tableau 7. Un peu plus de $60 \%(21 / 34)$ des programmes à caractère non professionnel sont regroupés sous la cote 6 . On n'en retrouve que deux sous les deux premières cotes, alors que plus de $60 \%$ des programmes à caractère professionnel (38/59) sont regroupés sous ces dernières. Cette fois-ci, cependant, ce sont les personnes diplômées des sciences appliquées et des sciences de la santé qui obtiennent le plus facilement un emploi relié à leur domaine d'études.

\subsection{Les résultats de la relance selon le sexe}

Au Québec, l'accessibilité des jeunes femmes aux études supérieures s'est améliorée au cours des dernières années. Au premier cycle, le nombre d'inscriptions féminines a crû dans tous les secteurs entre 1982 et 1989 , sauf dans ceux des arts et des lettres, où il a connu une faible diminution (Dupont et Léséleuc, 1991). En est-il de même de leur insertion professionnelle, comparativement à leurs homologues masculins?

Lorsque les deux groupes sont distingués de l'échantillon dans les résultats d'ensemble et après la correction des statistiques, on parvient aux résultats décrits dans le tableau 10 . Le test du $\chi^{2}$ a été utilisé.

Tableau 10 - Distribution, en 1989 au Québec, des personnes diplômées des universités québécoises en 1987

par catégories d'emploi, selon le sexe

\begin{tabular}{|c|c|c|c|c|}
\hline Catégories & Hommes & Femmes & Total & $x^{2}$ \\
\hline $\begin{array}{l}\text { Nombre de diplômés et diplômées } \\
\text { Diplômés et diplômées aux études } \\
\text { Diplômés et díplômées inactifs } \\
\text { Diplômés et diplômées sans emploi } \\
\text { Diplômés et diplômées en emploi } \\
\text { à temps plein } \\
\text { : relié au domaine } \\
\text { : non relié au domaine } \\
\text { : régulier } \\
\text { - temporaire } \\
\text { à temps partiel } \\
\text { - relié au domaine } \\
\text { - rón relié au domaine } \\
\text { régulier } \\
\text { temporaire }\end{array}$ & $\begin{array}{r}1184 \\
14,4 \% \\
0,9 \% \\
5,7 \% \\
79,0 \% \\
74,9 \% \\
60,7 \% \\
14,2 \% \\
61,8 \% \\
13,1 \% \\
4,1 \% \\
2,8 \% \\
1,3 \% \\
1,6 \% \\
2,5 \%\end{array}$ & $\begin{array}{r}12437 \\
12,1 \% \\
2,6 \% \\
7,9 \% \\
77,4 \% \\
66,4 \% \\
53,2 \% \\
13,2 \% \\
50,8 \% \\
15,6 \% \\
11,0 \% \\
7,8 \% \\
3,2 \% \\
4,5 \% \\
6,5 \%\end{array}$ & $\begin{array}{r}23 \mathbf{6 2 1} \\
13,2 \% \\
1,8 \% \\
6,8 \% \\
78,2 \% \\
70,6 \% \\
56,8 \% \\
13,7 \% \\
56,2 \% \\
14,3 \% \\
7,6 \% \\
5,3 \% \\
2,3 \% \\
0,3 \% \\
4,6 \%\end{array}$ & $\begin{array}{r}23,6 \\
94,55 \\
42,03 \\
* 1,93 \\
60,37 \\
58,33 \\
* * 4,31 \\
126,94 \\
25,96 \\
369,44 \\
278,55 \\
92,43 \\
166,35 \\
204,82\end{array}$ \\
\hline
\end{tabular}


Les résultats sont significatifs $\mathrm{p}=0,01$ pour toutes les catégories, sauf indications contraires. On observe donc que les diplômés sont plus nombreux que les diplômées à continuer leurs études, à détenir un emploi à temps plein régulier et relié à leur domaine d'études. Ces dernières sont plus nombreuses à détenir un emploi à temps partiel ou temporaire.

Idéalement, il aurait fallu ensuite calculer les deux indices décrits' auparavant de façon distincte pour chacun des deux sexes et pour chaque programme. Cela s'est malheureusement révélé impossible, car plusieurs des données nécessaires étaient absentes de la relance. Devant cette lacune, le nombre de diplômées et de diplômés disponibles sur le marché de l'emploi ou des études (TPR $+\mathrm{TPT}+\mathrm{TP}+\mathrm{ET}+\mathrm{CH}$ pour chacun des sexes) a été calculé pour chacun des programmes. À l'aide du coefficient de Pearson, il a alors été possible de vérifier s'il existe une relation entre la proportion d'hommes diplômés dans un programme et chacun des ratios. De même, la relation entre la proportion de femmes diplômées et les ratios a été calculée. Le coefficient mesure la covariance des deux distributions et possède ainsi une valeur prédictive, c'est-à-dire que plus il est élevé, plus sera élevée la probabilité qu'un programme de forte proportion d'un des deux sexes ait aussi un ratio élevé.

Tableau 11 - Relations entre les proportions d'hommes et de femmes au sein des programmes de premier cycle et les indices d'emploi à temps plein régulier (IPR) et d'emploi à temps plein relié au domaine d'études (IPL)

\begin{tabular}{lccc}
\hline Catégories & Hommes & Femmes & Total \\
\hline Nombre (disponibles) & $\mathbf{1 0 ~ 4 2 3}$ & $\mathbf{1 1 ~ 2 6 2}$ & \\
Pourcentage & $49,2 \%$ & $50,8 \%$ & \\
Moyenne des indices & & & 0,54 \\
IPL & & & 0,56 \\
- IPR & & & \\
Coefficient de corrélation (Pearson) & & & \\
- pourcentage pour chaque programme et IPL & 0,87 & 0,78 & \\
- pourcentage pour chaque programme et IPR & 0,91 & 0,75 & \\
\hline
\end{tabular}

Les résultats du tableau 11 permettent de constater que la relation est plus forte lorsque les personnes diplômées sont principalement de sexe masculin. Les programmes à forte proportion féminine offrent donc un pouvoir moindre à leurs diplômés et à leurs diplômées d'obtenir un em- 
ploi typique et relié à leur domaine. Les diplômées sont d'ailleurs prédominantes dans deux types de programmes: les programmes des sciences de la santé, dont les indices sont généralement élevés, (ergothérapie, optométrie, pharmacie) et certains programmes plus traditionnels aux indices plus faibles (enseignement et adaptation scolaire, service social, diététique et nutrition).

\section{Conclusion}

Il est possible de tirer les conclusions suivantes des résultats précédents.

Première conclusion. - La démonstration permet de réaliser l'impossibilité d'étudier l'insertion professionnelle des diplômées et des diplômés universitaires «en bloc», comme si ces derniers formaient un groupe social homogène quant à leur insertion. Il existe des distinctions importantes selon la famille de formation reçue, son caractère professionnel ou «académique» et selon le sexe. D'autres distinctions devront être ultérieurement faites. Par exemple, un survol d'autres données de la relance permet de réaliser que les personnes diplômées de certains programmes et qui sont occupées le sont toutes dans un ou deux secteurs d'emploi, alors que les personnes diplômées de certains autres programmes sont employées dans un grand nombre de secteurs variés. L'ajout d'une telle distinction pousserait à conclure que chaque programme universitaire et les secteurs d'emploi auxquels ses diplômées et ses diplômés ont accès doivent être des unités d'études distinctes. Tout effort de comprendre l'insertion professionnelle des universitaires dans leur ensemble ne peut que masquer la diversité de leur situation.

Deuxième conclusion. - De façon générale, les programmes à caractère professionnel offrent à leurs diplômées et à leurs diplômés un grand pouvoir d'obtention d'un emploi à caractère typique, de même qu'un emploi relié à leur domaine d'études. Les programmes à caractère plus académique offrent un pouvoir moindre. Les programmes aux indices les plus élevés sont surtout des formations des sciences de l'administration et des sciences appliquées. Ces constats militent en faveur de la nécessité de sensibiliser les étudiantes et les étudiants aux dangers de la «course aux diplômes». Si le but d'un étudiant ou d'une étudiante est 
d'augmenter ses chances d'obtenir un emploi typique, l'obtention d'un diplôme universitaire n'est pas nécessairement un moyen efficace. Toute décision devra prendre en considération le pouvoir fourni par un diplôme spécifique. En ce sens, une étude comparative de l'insertion professionnelle des diplômées et des diplômés des universités et des cégeps s'impose.

Troisième conclusion. - Si les femmes sont légèrement plus nombreuses que les hommes parmi les personnes diplômées, il existe des différences quant à leur situation réciproque sur le marché du travail. Les résultats de cette étude poussent à conclure à un plus grand pouvoir des hommes à obtenir un emploi typique et relié à leur domaine. Les programmes à prédominance masculine ont des indices plus élevés que les programmes à prédominance féminine. Il faut en conclure que pour les femmes, l'égalité de l'accès n'a pas encore conduit à l'égalité des résultats.

Quatrième conclusion. - Un peu moins de la moitié des diplômés universitaires sont sous-employés, quantitativement comme qualitativement. Un grand nombre de ressources humaines formées ne sont donc pas absorbées par le marché de l'emploi. Quant au potentiel de mobilité sociale de ces personnes diplômées, il faut conclure qu'elles n'ont pas encore les pieds sur le premier échelon. Leur situation sur le marché du travail se caractérise par l'emploi temporaire, à temps partiel ou le chômage. D'autres continuent leurs études, peut-être à cause des difficultés qu'ils rencontrent à trouver un bon emploi.

À la lumière de cette analyse, peut-on conclure que l'université québécoise ne réussit plus à remplir sa mission économique? Même si certains sont tentés de répondre par l'affirmative, il ne semble pas que ce soit là une conclusion soutenable. Ce qui semble beaucoup plus probable, c'est que la nature de la mission était irréaliste au départ. Les modifications majeures subies par les économies industrialisées durant les dernières quinze années font que l'expérience actuelle des personnes diplômées de l'université québécoise est partagée par les diplômées et les diplômés d'un très grand nombre d'autres pays (Sanyal, 1987; Coombs, 1985). On ne peut donc plus concevoir la formation supérieure comme un des moteurs du développement économique ni comme la garantie d'un 
bon emploi. La mission confiée à l'université était basée sur des prévisions fort optimistes de l'évolution du marché de l'emploi et une représentation plutôt simpliste de son fonctionnement, ce que Galbraith (1961) nommait avec justesse un «optimisme précaire». Bien que les universités aient participé à l'époque à cette forme particulière «d'autoséduction», d'autres acteurs qui ont été alors tout aussi enthousiastes et optimistes peuvent difficilement maintenant pointer du doigt des chiffres pour prouver l'échec de l'université.

Malheureusement, sur un plan social, dans la mesure ou la «foi» en la croissance a participé à élever les attentes de mobilité sociale d'une partie de la population et que la formation universitaire demeure liée, dans les représentations de plusieurs, à des emplois stables et intéressants, on constate aisément que l'université ne peut combler ces attentes pour un grand nombre de personnes diplômées.

Tout ceci milite en faveur d'une remise en question en profondeur du rôle de l'université dans la structure sociale, à la lumière des conséquences de la restructuration présente du marché de l'emploi.

\section{Références}

ANISEF, P., BERTRAND, M.A., HORTIAN, U. et JAMES, C.E. (1985).

L'accessibilité à l'enseignement postsecondaire au Canada, recension des ouvrages (Chapitre 5-L'accessibilité à l'enseignement postsecondaire au Québec). Ottawa : Secrétariat d'État.

AUDET, M. (1991).

Qu'advient-il des diplômés et des diplômées universitaires? La promotion de 1987. Québec : Les Publications du Québec.

BARIL, C. (1987).

Le succès professionnel des jeunes sur le marché du travail. Québec : Les Publications du Québec.

BERG, I. (1971).

Education and jobs : The great training robbery. Boston : Beacon Press.

BERNIER, L. (1986).

Tant qu'ils choisiront de vieillir. In Dumont, F. (éd.), Une société de jeunes? (p. 29 à 44). Québec : Institut québécois de la recherche sur la culture.

BERTCHERMAN, G., BÉDARD, M., CARON, C. et GERA, S. (1991).

Tertiarisation et polarisation des emplois. Ottawa : Conseil économique du Canada. 
BERTRAM, G. (1975).

L'éducation et la croissance économique. In Rocher, G. et Bélanger, P.W. (éd.), École et société au Québec (p. 153 à 174). Montréal : Hurtubise.

BUREAU DELA STATISTIQUE DU QUÉBEC (1984).

Les 15-29 ans, portrait statistique des jeunes par région. Québec : Les Publications du Québec.

CLARK, B. (1962).

Educating the expert society. San Francisco [CA] : Chandler.

COLEMAN, J.C. et HUSÉN, T. (1985).

Devenir adulte dans une société en mutation. Paris : OCDE.

COLEMAN, J.S. (1973).

Equality of opportunity and equality of results. Harvard Educational Review (février), 129-137.

COMMISSION ROYALE D'ENQUÊTE SUR L'ENSEIGNEMENT DANS LA PROVINCE DE QUÉBEC (1963).

Rapport, première partie: Les structures supérieures du système scolaire (Rapport Parent). Québec: Gouvernement du Québec.

COOMBS, P.H. (1985).

La crise mondiale de l'éducation. Bruxelles : De Bœck-Wesmaël.

DANDURAND, P., FOURNIER, M. et BERNIER, L. (1980).

Développement del'enseignement supérieur, classes sociales et luttes nationales au Québec. Sociologie et sociétés, 12(1), 101-131.

DORE, R. (1975).

The diploma disease. Berkeley et Los Angeles : Press of University of California.

DUCHASTEL, J. (1983).

Les enjeux sociaux d'une problématique de décroissance. In Pilon-Lê, L. et Hubert, A. (éd.), Les enjeux sociaux de la décroissance (p. 15 à 21). Montréal : Saint-Martin.

DUPONT, P. et LESÉLEUC, H. (1991).

Situation actuelle des inscriptions des femmes dans les universités québécoises. Carriérologie, 4(3), 17-35.

FORTIN, P. (1984).

Le chômage des jeunes au Québec : aggravation et concentration, 1966-1982. Relations industrielles, 39(3), 419-447.

FOURASTIE, J. (1972).

Faillite de l'université? Paris : Gallimard.

FRÉCHET, G. (1990).

Qualifications. In Langlois, S. et al. (éd.), La société québécoise en tendance, 1960-1990 (p. 169 à 172). Québec: Institut québécois de recherche sur la culture.

FULTON, O. (1984).

Needs, expectations and responses : New pressures on higher education. Higher Education, 13, 193-223. 
GALBRAITH, J.K. (1961).

L'ère de l'opulence (traduction française de «The Affluent Society»). Paris : Callman-Lévy.

GASKELL, J. (1991).

Education as preparation for work in Canada: Structure, policy and student response. In Ashton, D. et Lowe, G. (éd.), Making their way : Education, training and the labour market in Canada and Britain (p. 61 à 84). Toronto : University of Toronto Press.

GAUTHIER, M. (1991).

L'insertion de la jeunesse québécoise en emploi. Québec : Institut québécois de recherche sur la culture.

HARTMANN, J. (1988).

Au bord du précipice. Causes et conséquences de la diminution de l'emploi des jeunes en Europe. In Touraine, A. (éd.), Quel emploi pour les jeunes? Vers des stratégies novatrices (p. 53 à 99). Paris : UNESCO.

HOWES, G.R. et HOWES, L.S. (1982).

Concise dictionary of education. New York [NY] : Van Nostrand Reinhold.

HUSÉN, T. (1989).

Enseignement supérieur et stratification sociale : une comparaison internationale. Paris : UNESCO (IIPE).

LAHAYE, J. (1990).

Diplôme et accès aux diplômes dans les universités québécoises, 1976-1988. Québec : Direction générale de l'enseignement et de la recherche universitaire.

LAPERRIÈRE, A. (1982).

Quand on le peut... l'école pour éviter le pire. Revue internationale d'action communautaire, 8/48.

LEBRAS, H. (1983).

L'interminable adolescence ou les ruses de la famille. Le Débat, 25, 116-125.

LEDRUT, R. (1966).

Sociologie du chômage. Paris : PUF.

LOOSE, G. (1985).

Vocational education: Organization and sequences. In Husén, T. et NevillePostlethwaite, T. (éd.), The international encyclopedia of education (p. 5538 à 5546). Oxford : Pergamon.

MYLES, J., PICOT, G. et WANNELL, T. (1988).

Les salaires et les emplois au cours des années 1980. Évolution des salaires des jeunes et déclin de la classe moyenne. Document de recherche no 17. Ottawa : Direction des études analytiques, Statistiques Canada.

PAGEAU, D. et CHÉNARD, P. (1991).

De l'université au marché du travail : état de la situation des diplômées et des diplômés québécois du premier cycle. In Diambomba, M., Perron, M. et Trottier, C. (éd.), Les cheminements scolaires et l'insertion professionnelle des étudiants et des étudiantes de l'université (p. 133 à 250). Sainte-Foy: LABRAPS, Université Laval. 
RENAUD, J., BERNARD, P. et BERTHIAUME, M. (1980).

Éducation, qualification professionnelle et carrière au Québec. Sociologie et sociétés, 12(1), 23-52.

ROBERTS, K. (1988).

L'accès au marché du travail et l'orientation professionnelle : les nouveaux enjeux. Perspectives 18(4), 521-534.

RUMBERGER, R.W. (1981).

Overeducation in the US Labour Market. New York [NY] : Praeger.

SANYAL, B.C. (1987).

Higher education and employment : An international comparative analysis. New York [NY] : The Falmer Press.

TAYLOR, J. (1986a).

Comparing universities : Some observations on the first destination of new graduates. Higher Education Review, 19, 35-43.

TAYLOR, J. (1986b).

The employability of graduates: Differences between universities. Studies in Higher Education, 11(1), 17-27.

TAYLOR, J. et JOHNES, J. (1989).

An evaluation of performance indicators based upon first destination of college graduates. Studies in Higher Education, 14(2), 201-217.

TREMBLAY, D.G. (1989).

L'économie du travail : les réalités et les approches théoriques. Montréal : Saint-Martin.

TREMBLAY, D.G. et VAN SCHENDEL, V. (1982).

Le chômage des jeunes au Québec : un petit tour d'horizon. Revue internationale d'action communautaire, 8/48, 33-41.

Abstract. - Quebec universities were given, in the sixties, a mission that had two goals : to produce highly qualified workers and to allow social mobility to individuals. Because of the profound modifications that have taken place in the job market, a rethinking of this mission is in order. This article presents the position in the job market of degree graduates from ninety three different faculties with two scales: the index of regular full-time job (IPR) and the index of full-time job in line of specialization (IPL). For each ratio and program, results are grouped by programs and by category of education (professional or non-professional). The relationships between the indexes and the rate of graduates are examined as well. The results tend to prove the need for a rethinking of some of the foundations that led to give that mission to the universities of Quebec.

Resumen. - Durante los años sesenta se le confió a la universidad de Quebec una misión doble : desarrollar la mano de obra altamente calificada y permitir la mobilidad social de los individuos. Frente a las profundas modificaciones que ha sufrido el mercado del trabajo, se impone poner en tela de juicio dichas misiones. Este artículo presenta la posición relativa de los diplomados de noventa y seis programas de primer 
ciclo con respecto al mercado del empleo utilizando dos indicadores : el índice de empleo a tiempo completo regular (IPR) y el índice de empleo a tiempo completo ligado al campo de estudios (IPL). Para cada índice y cada programa, los resultados se agrupan según la familia de programas y el tipo de formación (profesional o no profesional). La relación entre los índices y la proporción de universitarios diplomados de diversos programas se examina también. Los resultados conducen a poner en duda ciertos postulados que condujeron al enunciado de la misión de la universidad de Quebec.

Zusammenfassung. - Im Laufe der sechziger Jahre bekamen die Quebeker Universitäten einen zweifachen Auftrag : hochqualifizierte Arbeitskräfte hervorzubringen und die soziale Beweglichkeit der Individuen zu ermöglichen. Angesichts der tiefgreifenden Veränderungen, die der Arbeitsmarkt gegenwärtig erfährt, muß dieser Autrag neu durchdacht werden. Dieser Artikel präsentiert die relative Position der Hochschulabsolventen und -absolventinnen von 93 Studienplänen des Grundstudiums auf dem Stellenmarkt und dies mit Hilfe von zwei Indikatoren : dem Index derregulären Vollzeitbeschäftigung (IPR) und dem Index der Vollzeitbeschäftigung im Studienbereich (IPL). Für jeden Index und jedes Programm werden die Ergebnisse je nach Programmgruppe oder Ausbildungstyp (gewerblich oder nicht-gewerblich) zusammengestellt. Die Beziehung zwischen den Indexen und der Proportion der Hochschulabsolventen/-absolventinnen der verschiedenen Studienpläne wird ebenfalls untersucht. Die Ergebnisse führen zur Infragestellung mancher Postulate, die der Erteilung des Auftrages an Quebecs Universitäten zugrunde lagen. 